Itinéraires Itinéraires

Littérature, textes, cultures

\title{
Andrew J. Webber, The European Avant-Garde, Cultural History of Literature
}

\section{Emmanuel Guy}

\section{OpenEdition}

\section{Journals}

Édition électronique

URL : http://journals.openedition.org/itineraires/565

DOI : 10.4000/itineraires.565

ISSN : 2427-920X

Éditeur

Pléiade

\section{Édition imprimée}

Date de publication : 1 novembre 2009

Pagination : 188-190

ISBN : 978-2-296-10115-9

ISSN : 2100-1340

\section{Référence électronique}

Emmanuel Guy, «Andrew J. Webber, The European Avant-Garde, Cultural History of Literature », Itinéraires [En ligne], 2009-3 | 2009, mis en ligne le 27 juin 2014, consulté le 22 septembre 2020. URL : http:// journals.openedition.org/itineraires/565; DOI : https://doi.org/10.4000/itineraires.565

\section{cc)}

Itinéraires est mis à disposition selon les termes de la licence Creative Commons Attribution - Pas d'Utilisation Commerciale - Pas de Modification 4.0 International. 
Andrew J. Webber, The European Avant-Garde, Cultural History of Literature, Cambridge, Cambridgeshire, Polity Press, 2004.

Andrew J. Webber, auteur d'une thèse intitulée Sexuality and the Sense of Self in the Works of Georg Trakl and Robert Musil (Londres, 1990), et d'une étude sur la figure du Double dans la littérature romantique allemande (The Doppelgänger, Oxford, Clarendon Press, 1996) propose dans son dernier ouvrage d'appliquer son intérêt théorique pour la psychanalyse des productions artistiques et littéraires à un nouveau corpus, les avant-gardes européennes de la première moitié du $\mathrm{Xx}^{\mathrm{e}}$ siècle.

Webber consacre son premier chapitre à une introduction théorique. Il y précise la teneur de son projet : proposer une étude comparatiste, et avant tout littéraire, des manifestations " classiques " de l'avant-garde historique. Se référant plaisamment à la pipe de Magritte, l'auteur dit cependant que son livre «sera » et « ne sera pas de l'histoire culturelle». Le lecteur, lui, sera perplexe, à n'en pas douter. Il le restera peut-être avec la présentation d'une allégorie critique récurrente dans tout l'ouvrage, l'Angelus Novus de Paul Klee; Walter Benjamin en faisait la figure d'une méthode, l'approche historique, Webber, qui le cite, en fait la figure de son objet d'étude, l'avant-garde. Parce qu' « il regarde en arrière mais se projette en avant », cet ange va servir à Webber de métaphore filée d'une idée somme toute fort commune : la relation des avant-gardes au passé est bien plus complexe que ce que suggère leur rhétorique du renouvellement radical. Certes. On attendra impatiemment de voir comment Webber se positionne historiographiquement : la partie consacrée aux « théories et histoires de l'avant-garde» ne comporte, en fait de théories et d'histoires, qu'un seul ouvrage proprement relatif au sujet, le désormais canonique Théorie de l'Avant-garde de Peter Brüger (1974). Webber ne cache pas sa franche préférence pour la théorie et les théorisations. Il adresse à Bürger des griefs assez traditionnels, mais les conclusions qu'il en tire pour justifier son approche se situent à l'opposé des conclusions traditionnelles. Loin de se cantonner à un corpus plus limité que son prédécesseur et à n'en proposer qu'une approche sciemment relative, Webber entend aborder l'avant-garde dans son ensemble et montrer la multiplicité des théories qu'on peut lui appliquer. Le projet est ambitieux, et on regrettera que l'auteur n'ait pas mis son goût pour la théorie au service d'une mise en perspective de la notion, et d'une problématisation de ses diverses manifestations. Si l'objet de l'étude ne bénéficie pas d'une définition problématisée, la méthode présentée n'en reste pas moins prometteuse : Webber annonce un mélange de critique matérialiste marxiste et de psychanalyse freudienne et post-freudienne. Il se réclame ici de Benjamin qu'il relit au prisme de Frederic Jameson et de sa notion d' « inconscient politique » : à la suite du philosophe de Francfort, Webber entend décrypter l' " inconscient historique » dans les " vestiges de la culture matérielle». On est moins convaincu en revanche lorsque Webber définit l'objectif de cette méthode comme une tentative de "retourner la stratégie du choc typique de l'avant-garde contre ses objets, pour y surprendre les identités, les caractéristiques et les relations jusqu'ici négligées » (c'est nous qui soulignons). En réalité, l'étude de Webber se place avant tout dans une perspective psychanalytique, l'approche matérialiste au sens strict y étant quasi absente ou 
alors seulement dans une confusion jamais éclaircie entre avant-garde artistique et avant-garde politique.

Le reste de l'ouvrage entend mettre en application la méthodologie exposée en abordant successivement les manifestes historiques, quelques chefs-d'œuvre de Magritte ou Duchamp, quatre écrits sur la ville, des séquences du cinéma d'Eisenstein et le théâtre d'Artaud, et finalement, une demi-douzaine de fictions narratives d'auteurs plus ou moins notoirement d'avant-garde, tels que Virginia Woolf, Reiner Maria Rilke, Aragon, Kafka, Gertrude Stein ou André Breton. On sera impressionné de la variété et de la maîtrise des exemples choisis par Webber, dans des aires culturelles, des médiums et des courants esthétiques divers.

« Ne pourrait-on soutenir la thèse que l'intérêt d'un texte critique réside presque exclusivement dans sa méthode? », ainsi commençait l'étude de Rosalind Krauss sur les avant-gardes. Ce qui fait l'intérêt de l'étude de Webber n'est ni le choix des textes abordés, tous très connus, ni les conclusions auxquelles il arrive, assez convenues, mais peut-être le détail de sa méthode, les méandres de ses analyses qui révèlent parfois d'heureuses intuitions ou de riches trouvailles.

L'approche psychanalytique des œuvres de l'avant-garde n'est pas neuve : Margaret Cohen a montré à propos d'André Breton la pertinence et la légitimité d'une telle approche dans Profane Illuminations (1993) et David Macey a étudié l'importance du surréalisme chez Lacan (voir Lacan in Context, 1988). Webber n'évoque pas ces prédécesseurs, mais systématise l'approche psychanalytique à l'occasion de nombreux close readings. Au sujet des manifestes par exemple, il propose une relecture stimulante de textes fort connus par ailleurs. Il se montre un lecteur attentif et inventif dans la lecture de ces textes canoniques que sont le manifeste DADA de Tzara, le Waste Land d'Eliot ou Fräulein Else de Schnitzler (encore qu'il soit difficile pour ce texte, et Webber en convient, de parler d'avant-garde historique). De même, il approche avec une acuité visuelle et une sensibilité esthétique certaines des œuvres comme le LHOOQ de Duchamp, Le Viol de Magritte ou le Da-Dandy de Hannah Höch. Il est très délicat de résumer les analyses précises de Webber, tant l'auteur nous met immédiatement aux prises avec le détail, la lecture de près, sans préalable contextuel ou résumé inaugural. Chaque chapitre réunit une poignée d'explications de détails autour d'une conclusion, parfois décevante par sa banalité : le chapitre sur la ville conclut que le dénominateur commun des textes abordés n'est autre que la tension entre le choix d'une forme épique, donc traditionnelle, et le recours à des procédés poétiques proprement avant-gardistes, donc révolutionnaires. Selon Webber, cette tension donne à ces écrits une tonalité mélancolique, expression de la voix du poète perdu dans la cité moderne. Webber consacre ensuite un chapitre aux arts de la performance où il étudie sous l'angle de l'effet de distanciation brechtien le théâtre d'Artaud et les effets de montage du cinéma d'Eisenstein. Il clôt son analyse sur l'étude de romans modernistes, dont il cherche à extraire ce qu'il appelle la « teneur avant-gardiste »; on est très gêné, dans tout le chapitre, par l'habitude tenace de Webber de ne pas problématiser les catégories de l'histoire littéraire auxquelles il se réfère. 
Ainsi, sous le titre ambitieux, L'Avant-Garde européenne, dans une collection explicitement consacrée à l'histoire culturelle de la littérature, ce livre rassemble surtout une vaste collection d'explications de textes, d'interprétations de tableaux ou de séquences filmées, reliées entre elles par une intarissable fièvre théorique. Webber ne s'intéresse ni aux conditions de productions, ni au contexte historique, ni aux carrières, ni aux transferts culturels entre avant-gardes, mais seulement à des fragments extraits de tel ou tel artiste dit «d'avant-garde ». Son intérêt se porte avant tout sur la théorie, il recourt à des termes fortement chargés de sens, tels que le matérialisme, la psyché, l'inconscient, mais en fait un usage si extensif qu'on finit un peu perdu entre une métaphore filée à visée rhétorique, et un jeu de mot faisant office de transition entre les éléments de son corpus.

D'une lecture suivie très exigeante, l'ouvrage saura peut-être retenir l'attention de lecteurs intéressés par l'application des théories freudiennes aux productions littéraires et artistiques, et éveiller l'intérêt du lecteur curieux de découvrir une approche originale de textes et d'œuvres désormais canoniques.

\section{Emmanuel Guy \\ Université Paris $13-C E N E L$}

Laura Doyle and Laura Winkiel (dir.), Geomodernisms. Race, Modernism, Modernity, Bloomington, Indiana University Press, 2005, 354 p.

Cette publication, qui regroupe les contributions d'une vingtaine de chercheurs, est le fruit de différents séminaires organisés par The Modernist Studies Association Conference, de 2000 à 2003. Échanges et débats nombreux qui ont contribué, sans doute, à inscrire l'ouvrage dans une perspective de nécessaire dynamisme dans la manière de repenser les concepts et de les réagencer pour aborder la question du « modernisme » sous des angles différents.

Ce concept de « modernism », central dans l'ouvrage, donne lieu à de nombreuses mises en perspective et est le premier à passer au crible de la critique, qui choisit, tout d'abord, de ne le décliner qu'au pluriel, tant il semble en effet difficile de pouvoir postuler un seul et unique modernisme (ainsi qu'une seule et unique modernité) et tant cette notion semble impossible à circonscrire, ne cessant de se dérober et se transformant en permanence selon les époques et les points de vue.

Le lecteur, ainsi confronté d'emblée à l'impossibilité de travailler à partir d'un concept unique, découvre d'autres partis pris nécessaires :

To emplace modernism in this way - écrivent ainsi Laura Doyle et Laura Winkiel dans leur introduction - to think, rather, in terms of interconnected modernisms - requires a rethinking of periodization, genealogies, affiliations and forms. To some degree, this rethinking estranges the category of modernism itself. The term modernism breaks open, into something we call geomodernisms, which signals a locational approach to modernisms' engagement with cultural and political discourses of global modernity. (p. 3) 